\title{
SOLVING ASSET PRICING MODELS WHEN THE PRICE-DIVIDEND FUNCTION IS ANALYTIC
}

\author{
By OVIDiu L. CALIN, Yu Chen, ThOMAS F. Cosimano, \\ AND AlEX A. HimONAS ${ }^{1}$
}

\begin{abstract}
We present a new method for solving asset pricing models, which yields an analytic price-dividend function of one state variable. To illustrate our method we give a detailed analysis of Abel's asset pricing model. A function is analytic in an open interval if it can be represented as a convergent power series near every point of that interval. In addition to allowing us to solve for the exact equilibrium price-dividend function, the analyticity property also lets us assess the accuracy of any numerical solution procedure used in the asset pricing literature.
\end{abstract}

KEYWORDS: Analyticity, asset pricing, solving, numerical analysis.

\section{INTRODUCTION}

RESEARCHERS IN FINANCIAL ECONOMICS have sought solutions for asset pricing models in the space of bounded and continuous functions ever since the seminal work of Lucas (1978). ${ }^{2}$ This paper seeks solutions to asset pricing problems in the more regular space of analytic functions. A function $f(x)$, defined on $\Omega$ an open subset of $\mathbb{R}$, is analytic at a point $x_{0} \in \Omega$ if it can be expressed as a convergent power series in some neighborhood of $x_{0}$. If $f(x)$ is analytic for every $x_{0} \in \Omega$, then it is analytic in $\Omega .^{3}$ We follow this strategy because of several observations concerning most applied asset pricing models: First, the pricing kernel and dividend process are analytic functions on a given set. Second, these components of the asset pricing models are incorporated into an integral equation that maps the unknown future price-dividend function into the current price-dividend function. Finally, the equilibrium price-dividend function is the solution to this integral (functional) equation that preserves the properties of the underlying function. These observations lead us to the con-

\footnotetext{
${ }^{1}$ The authors would like to thank Professor Stefano Athanasoulis for numerous discussions during the early stages of this work and for the simulation implemented in Section 3. We would like to thank seminar participants at Oxford University Said School of Business, the University of Notre Dame, the 2003 Summer Econometric Society Meetings, and the 2003 Stanford Institute for Theoretical Economics Workshop on Computational Methods and Applications in Economics for helpful comments. Discussions with Sebatien Cottrell, Thomas Gresik, Kenneth Judd, and Ali Khan helped in the preparation of this work. We also benefited from valuable comments of two anonymous referees and the editor. They helped us improve this paper significantly. Any remaining errors are our responsibility. Tom Cosimano received financial support from the Center for Research in Financial Services Industry at the University of Notre Dame.

${ }^{2}$ See Altuğ and Labadie (1994) for a summary of this work.

${ }^{3}$ See Rudin (1974, pp. 212-249).
} 
clusion that the price-dividend function is analytic for several popular asset pricing models. ${ }^{4}$

Our procedure consists of the following steps: First, we rewrite the Euler equation for the asset pricing model as a functional equation and identify the space (see Definition 1) on which the price-dividend function is a well-defined function of one state variable, i.e., dividend growth. By well defined we mean that the conditions of a fixed point theorem hold, thus a continuous and integrable equilibrium price-dividend function exists and is unique. Second, we apply a change of variable so that the future price-dividend function is dependent on only the random shock to the state variable and independent of the current state variable. These steps make the integrand for the integral equation the product of a continuous and integrable function of the random variable and an analytic function; see Equation (6). Third, we prove that the price-dividend function is infinitely differentiable in the open interval where the function is well defined. Fourth, we complexify the integral, i.e., in the integral for the asset pricing model we replace the real state variable, $x$, with its complex counterpart, $z=x+i y$. Finally, using the Lebesgue dominated convergence theorem (DCT) we prove that the complex integral (see Equation (12)) is analytic in the state variable. Thus, the analytic properties of the pricing kernel transfer to the function defined by the integral, so that the pricedividend function is analytic on a given set.

We demonstrate this method for Abel's (1990) asset pricing model in the Mehra and Prescott (1985) case and the external habit case. ${ }^{5}$ We feel that solving these two cases allows the reader to see how the method works and how it could be generalized to handle more complicated asset pricing models. ${ }^{6}$ Observe that the second and third steps in our procedure only require the utility function and dividend process to be analytic on the set where the

\footnotetext{
${ }^{4}$ The known solutions to the price-dividend function in asset pricing models with constant absolute (CARA) and relative (CRRA) utility function are all analytic. Wang (1994) and Athanasoulis and Shiller (2001) provide recent examples for CARA utility, while Campbell (1986), Labadie (1989), Burnside (1998), Birdarkota and McCulloch (2003), and Tsionas (2003), among others, provide solution for models with CRRA utility. Our method finds the solutions for analytic utility functions that offer closed forms for a wider class of probability distributions for the state variable.

${ }^{5}$ While we have not included the internal habit case, the earlier version of this paper demonstrated that this model is analytic as well. See Athanasoulis, Calin, Cosimano, and Himonas (2003) (ACCH). A copy of this paper and computer programs for the numerical analysis may be obtained at http://www.nd.edu/ tcosiman/. The parameterization that includes internal habit persistence in Abel's model is problematic: it leads to negative marginal utility for some values of consumption growth with internal habit persistence. Consequently, this case demonstrates that this procedure works only when the integral equation is well defined.

${ }^{6}$ Our method also applies to other asset pricing models such as, for example, Epstein and Zin (1989, 1990, 1991), Campbell and Cochrane (1999), Cecchetti, Lam, and Mark (1990, 2000), and Constantinides and Duffie (1996), among several others. The additional complexity of those models makes the proofs more involved, although this methodology can handle these cases. For example, Chen, Cosimano, and Himonas (2003) applies this methodology to the Campbell
} 
price-dividend function is well defined. Consequently, this method can handle many different types of analytic dividend processes and utility specifications. Thus, our procedure is general enough to apply to many of the asset pricing problems found in the literature.

Knowing the set on which the price-dividend function is analytic is important for the evaluation of the accuracy of numerical solutions to asset pricing models. ${ }^{7}$ In each case of Abel's asset pricing models we know that the pricedividend ratio is a convergent power series on the set where the price-dividend function is analytic. In addition, we know the radius of convergence for this power series. This allows us to use a high-order Taylor polynomial to represent the price-dividend function in the neighborhood of a point where the pricedividend function is analytic. The error in the Taylor approximation can be made smaller than machine epsilon, i.e., less than $10^{-16}$, as long as the size of the neighborhood of the point is sufficiently less than the radius of convergence or there are a sufficient number of coefficients for the Taylor series. ${ }^{8}$ This numerical solution allows us to establish the accuracy of a state of the art numerical method for solving these asset pricing models.

We begin, in the next section, by outlining Abel's asset pricing model. We follow this by identifying the space where the price-dividend function is well defined. Next, we prove that the price-dividend function is infinitely differentiable. We then summarize the properties of analytic functions followed by the proof that the price-dividend function is analytic. We conclude this section by computing and analyzing the error of a polynomial approximation to the price-dividend function motivated by the analyticity of the solution. Section 3 provides the numericalcomparisons between our numerical approximation and the Gauss quadrature procedure for Abel's asset pricing model. We conclude the paper in Section 4.

\section{SOLVING ASSET PRICING MODELS}

The classic case for the equity premium puzzle is the Mehra and Prescott (1985) model. ${ }^{9}$ The Mehra and Prescott model is subsumed by Abel's (1990)

and Cochrane model. We cannot apply this methodology to asset pricing models with the Kalman filter, as in Wang (1994), or with an ARCH model, as in Engle (1982), since these models have two state variables. We plan to address these problems in future research.

${ }^{7}$ The perturbation method (see Judd (1996, 1998), Judd and Guu (2001), and Jin and Judd (2002)) is based on the solution being analytic; however, this procedure does not identify the set where the price-dividend function is analytic. As a result, the accuracy of the solution cannot be identified. This explains why Collard and Juillard (2001) compares the perturbation method results with Burnside's (1998) explicit formula for Mehra and Prescott's model.

8"Machine epsilon" is the largest $\epsilon$ so that the computer cannot tell the difference between $a$ and $a \pm \epsilon$ for a given number $a$. See Judd (1998, p. 30).

${ }^{9}$ See Mehra and Prescott (2003) for an update on the equity premium puzzle literature. 
model, which also includes habit persistence, both internal and external. In this section we first prove the unique equilibrium price-dividend function is continuous, integrable, and infinitely differentiable. We go on to prove the theorem that the price-dividend function for Abel's model is analytic when the pricedividend function is well defined. The price-dividend function is well defined as long as the integral equation is a mapping from a vector space of integrable functions to the same vector space and it yields a unique integrable solution.

\subsection{Abel's Asset Pricing Model}

There are four assumptions to the models of Mehra and Prescott or Abel: (1) There is a representative agent that has an analytic utility function on a given set, (2) financial markets are complete, (3) financial markets are frictionless, and (4) individuals are free to trade the risky stock and one period bonds which are in zero net supply. Models with these assumptions and some variant of them are solved for numerically in the literature, and we wish to get the actual analytic solution to the asset prices here. ${ }^{10}$

Abel's model has the following specific structure. Individuals have the utility function

$$
\frac{\left[c_{t} / v_{t}\right]^{1-\gamma}}{1-\gamma}
$$

where $v_{t}=\left[c_{t-1}^{\rho} C_{t-1}^{1-\rho}\right]^{\alpha}, c_{t-1}$ is the consumer's own consumption in period $t-1$, and $C_{t-1}$ is aggregate per capita consumption in period $t-1$. The parameters have the restrictions $\gamma>0, \alpha \geq 0$, and $\rho \geq 0$. Abel parameterizes the model by setting $\alpha$ and $\rho$ to 0 or 1 , depending on the case. Note that when $\alpha=0$ we have Mehra and Prescott's model. When $\alpha=1$ and $\rho=0$ we are in the case of relative consumption of catching up with the Joneses. This case is also called external habit in which the habit is external to the individual's choice. When $\alpha=1$ and $\rho=1$ we are in the internal habit case and individuals own lagged consumption affects their choice of consumption.

The only source of real income is the dividend from the risky security so that $c_{t}=C_{t}=D_{t}$. The dividend process for the risky security is

$$
D_{t+1}=D_{t} \exp \left[x_{0}+\phi x_{t}+\nu_{t+1}\right],
$$

where $\nu \sim \operatorname{NIID}\left(0, \sigma^{2}\right)$, so that $x_{t}$ is the current continuously compounded growth rate of the dividend and $x_{0}$ is the constant growth rate per period so that dividend growth follows an $\mathrm{AR}(1)$ process subject to a normally distributed random shock.

\footnotetext{
${ }^{10}$ With constant relative risk averse utility, this model has been solved for by Campbell (1986), Labadie (1989), Burnside (1998), Birdarkota and McCulloch (2003), and Tsionas (2003).
} 
Abel shows that the Euler equation for equity is

$$
\frac{P_{t}}{D_{t}}=E_{t}\left[M_{t+1} \frac{D_{t+1}}{D_{t}}\left(1+\frac{P_{t+1}}{D_{t+1}}\right)\right]
$$

where the intertemporal rate of substitution is given by

$$
M_{t+1}=\beta\left(\frac{D_{t+1}}{D_{t}}\right)^{-\gamma}\left(\frac{v_{t+1}}{v_{t}}\right)^{\gamma-1} \frac{H_{t+2}}{E_{t}\left(H_{t+1}\right)} .
$$

We also have $H_{t+2}=1-\beta \alpha \rho\left(D_{t+2} / D_{t+1}\right)^{1-\gamma}\left(D_{t+1} / D_{t}\right)^{(-\alpha(1-\gamma))}, \alpha \geq 0, \rho \geq 0$, and $v_{t+1}=D_{t}^{\alpha}$.

Examination of the Euler equation (3) and intertemporal rate of substitution (4) reveals that they are homogeneous of degree 1 in the stock price, current dividends, and next period's dividends. Consequently, we seek a solution to the equilibrium price-dividend function, $P(x)$, which is dependent on the current dividend growth, $x$. Here we drop the subscript for time since we seek a function of current dividend growth. We work with the price-dividend ratio rather than expected returns although we are able to convert these price-dividend ratios into expected returns and compare the results in the literature to ours.

By substituting the intertemporal rate of substitution (4) and dividend process (2) into the Euler equation for equity (3), we derive the fundamental integral equation for Abel's asset pricing model:

$$
\begin{aligned}
& P(x)= \frac{1}{\sqrt{2 \pi} \sigma} \frac{K_{0} \exp \left[K_{1} x\right]}{1-K_{2} \exp \left[K_{1} x\right]} \\
& \times \int_{-\infty}^{\infty} \exp \left[-\frac{1}{2 \sigma^{2}}\left[\nu-\sigma^{2}(1-\gamma)\right]^{2}\right] \\
& \quad \times\left[1-K_{2} \exp \left[K_{1}\left(x_{0}+\phi x+\nu\right)\right]\right] \\
& \quad \times\left[1+P\left(x_{0}+\phi x+\nu\right)\right] d \nu .
\end{aligned}
$$

Here we define $K_{0}=\beta \exp \left(x_{0}(1-\gamma)+\frac{\sigma^{2}}{2}(\gamma-1)^{2}\right), K_{1}=(1-\gamma)(\phi-\alpha)$, and $K_{2}=\alpha \rho K_{0}$.

\subsection{Existence and Uniqueness of Price-Dividend Function}

The proof of existence and uniqueness of the price-dividend function must confront two problems with the fundamental integral equation for Abel's asset pricing model. The first problem recognized by Abel is the possibility of a negative marginal utility of consumption as reflected in the term $1-K_{2} \exp \left[K_{1} x\right]$. 
For the Mehra-Prescott case and the external habit case this does not present an issue since $K_{2}=0$. However, it does present a problem for the internal habit case. For the parameters considered here the marginal utility would be negative for dividend growth larger than 1.2 standard deviations. ${ }^{11}$ This condition is violated by the assumption of a normal distribution for the dividend growth shock. Limiting our attention to the Mehra and Prescott and external habit cases allows us to avoid this issue, and thus to focus more clearly on the issue of when the price-dividend function is analytic. The second problem deals with the existence of a bounded solution. The price-dividend function is not bounded given the normal distribution for dividend growth and $K_{1}>0 .{ }^{12} \mathrm{In}$ stead of looking for a solution in the vector space of bounded functions, we examine the following vector space where the integral in (6) exists.

DEFINITION 1: Let $\mathcal{S}$ denote the real vector space that consists of all continuous functions $f$ satisfying $|f(x)| \leq M_{f} \exp \left[k_{f}|x|\right]$ for some positive constants $M_{f}$ and $k_{f}$, where the constants may depend on $f$.

Within the space $\mathcal{S}$ we seek the solution to the integral equation

$$
\begin{aligned}
P(x)= & K_{0} \exp \left[K_{1} x\right] \\
& +\frac{K_{0} \exp \left[K_{1} x\right]}{\sqrt{2 \pi} \sigma} \int_{-\infty}^{\infty} \exp \left[-\frac{1}{2 \sigma^{2}}[s-\psi(x)]^{2}\right] P(s) d s .
\end{aligned}
$$

Here, we modify the integral equation (5) by making the change of variable $s=x_{0}+\phi x+\nu$ so that $\psi(x)=\phi x+x_{0}+\sigma^{2}(1-\gamma) \cdot{ }^{13}$ This change of variable is helpful in the proof of differentiability and when the price-dividend function is analytic, since we never have to explicitly differentiate the price-dividend function in the integrand.

First we establish that the second term on the right-hand side of (6) defines a linear transformation from the space $\mathcal{S}$ into $\mathcal{S}$ (see Lemma 4). Then we show

\footnotetext{
${ }^{11}$ In ACCH (2003) we did examine internal habit cases in which the coefficient of relative risk aversion was near 1 so that the random shock could be less than 3 standard deviations.

${ }^{12}$ This problem explains why it is generally necessary to bound the support of the random shocks to dividend growth. See, for example, Altuğ and Labadie (1994). There are several ways to address this problem: (1) Truncate the distribution for dividend growth; (2) change the integral equation by assuming the price-dividend function is fixed above a given level since higher levels of dividend growth are never observed; (3) the procedure adopted here. The first procedure is tedious since we have to keep track of the change of limits of integration in all the proofs. The second procedure was used in the earlier version of this paper. We adopted the current procedure since it yields the largest radius of convergence.

${ }^{13}$ Notice that $x$ is the state variable. In addition, $s$ is the variable of integration, i.e., the random disturbance to the state variable.
} 
that this linear transformation has a unique fixed point in $\mathcal{S}$, as long as

$$
\begin{aligned}
K_{5}= & K_{0}\left[1+2 \Phi\left(\frac{\left|\phi K_{1}\right| \sigma}{1-|\phi|}\right)\right] \\
& \times \exp \left(x_{0} K_{1}+\frac{\sigma^{2}(1-\gamma)^{2}(\phi-\alpha)(2+\phi-\alpha)}{2}\right. \\
& \left.\quad+\frac{\phi^{2} K_{1}^{2} \sigma^{2}}{2(1-|\phi|)^{2}}+\frac{\left|\phi K_{1} \theta\right|}{1-|\phi|}\right)<1,
\end{aligned}
$$

where $\theta=x_{0}+\sigma^{2}(1+\phi-\alpha)(1-\gamma)$ and $\Phi(x)=\frac{1}{\sqrt{2 \pi}} \int_{0}^{x} \exp \left[-s^{2} / 2\right] d s$ for all $x \in \mathbb{R}^{14}$ Thus, we have the following result, whose proof is included in the Appendix for completeness sake. The Appendix is available at the Supplementary Material web site for Econometrica.

Proposition 1: If $K_{5}<1$, where $K_{5}$ is given by (7), then there is a unique solution to the equation (6) in the vector space $\mathcal{S}$.

This result corresponds to the typical analysis of asset pricing models in that a solution is found in a general space of continuous functions, however, we modify the analysis by looking in the space $\mathcal{S}$ in which functions are integrable with respect to the measure $\exp \left[-\frac{1}{2 \sigma^{2}} x^{2}\right] d x$ rather than bounded functions. Next, we show differentiability of the price-dividend function.

\subsection{Differentiability of Price-Dividend Function}

While the existence and uniqueness of the price-dividend function are well known, there is only limited information about the differentiability of the price-dividend function for general models. Most analysis on differentiability deals with the policy and value functions from a generic dynamic programming problem. ${ }^{15}$ By focusing on a specific integral equation, in our case the one from Abel's asset pricing model, we can obtain much more information about the differentiability of the price-dividend function.

PROPOSITION 2: If $K_{5}<1$, then the solution to the integral equation (6) for the price-dividend function is infinitely differentiable for all dividend growth, $x \in \mathbb{R}$.

\footnotetext{
${ }^{14}$ Here, $2 \Phi\left(\left(\left|\phi K_{1}\right| \sigma\right) /(1-|\phi|)\right)$ is the probability that dividend growth is in the interval $\left[-\left(\left|\phi K_{1}\right| \sigma\right) /(1-|\phi|),\left(\left|\phi K_{1}\right| \sigma\right) /(1-|\phi|)\right]$. For the parameter values considered below $0<$ $K_{5} \leq .9304$ in the Mehra-Prescott case and $0<K_{5} \leq .9782$ in the external habit case.

${ }^{15}$ Blume, Easley, and O'Hara (1982) develop conditions for policy and value functions to have an $r$ th-order derivative. In addition, Santos $(1991,1992,1993,1994,1999)$ finds conditions for the existence of the first two derivatives of general policy and value functions.
} 
PROOF: It suffices to show that for any positive integer $n$, we always have

$$
\begin{aligned}
& \frac{d^{n}}{d x^{n}} \int_{-\infty}^{\infty} P(s) \exp \left[-\frac{1}{2 \sigma^{2}}[s-\psi(x)]^{2}\right] d s \\
& \quad=\int_{-\infty}^{\infty} P(s) \frac{\partial^{n}}{\partial x^{n}} \exp \left[-\frac{1}{2 \sigma^{2}}[s-\psi(x)]^{2}\right] d s
\end{aligned}
$$

By the definition of $\mathcal{S}$ we can find positive constants $M$ and $k$ with $|P(x)| \leq M \exp [k|x|]$. The $n$th partial derivative of $\exp \left[-\frac{1}{2 \sigma^{2}}[s-\psi(x)]^{2}\right]$ with respect to $x$ is expressible as

$$
\exp \left[-\frac{1}{2 \sigma^{2}}[s-\psi(x)]^{2}\right] \sum_{i=0}^{n} f_{i}(x) s^{i}
$$

where the $f_{i}$ are polynomials. It is easy to check that $s^{i} \leq \exp [i|s|]$ for any integer $i \geq 0$, where we regard $s^{0}=1$ for all $s \in \mathbb{R}$. Given any real numbers $a \leq b$, we have

$$
\begin{aligned}
& \left|\int_{a}^{b} P(s) \frac{\partial^{n}}{\partial x^{n}} \exp \left[-\frac{1}{2 \sigma^{2}}[s-\psi(x)]^{2}\right] d s\right| \\
& \quad \leq M \sum_{i=0}^{n}\left|f_{i}(x)\right| \int_{a}^{b} \exp \left[-\frac{1}{2 \sigma^{2}}[s-\psi(x)]^{2}+(i+k)|s|\right] d s .
\end{aligned}
$$

A lemma proved in the Appendix shows that the improper integrals $\int_{-\infty}^{\infty} \exp \left(-\frac{1}{2 \sigma^{2}}[s-\psi(x)]^{2}+(i+k)|s|\right) d s$ are all uniformly convergent when $x$ lies in any bounded closed interval. Thus, the integral $\int_{-\infty}^{\infty} P(s) \frac{\partial^{n}}{\partial x^{n}} \exp \left(-\frac{1}{2 \sigma^{2}} \times\right.$ $\left.[s-\psi(x)]^{2}\right) d s$ is uniformly convergent on any bounded closed interval for $x$. By Theorem 58 in Kaplan (1956) the solution to (6) is infinitely differentiable for $x \in \mathbb{R}$.

Q.E.D.

\subsection{Analyticity of Price-Dividend Function}

We now present a summary of the properties of an analytic function. ${ }^{16}$ A function $f(x)$ is (real) analytic in an open interval $(a, b)$ if it can be ex-

${ }^{16}$ This discussion is based on Chapter 10 of Rudin (1974). For this discussion we follow mathematical convention, and use $f$ and $x$ to represent a generic function and variable, respectively. This notation makes it easier to read the original source. 
pressed as a convergent power series near every point of the interval $(a, b)$. More precisely, $f(x)$ is analytic in $(a, b)$ if for every $x_{0} \in(a, b)$ there is a positive real number $r$, which may depend on $x_{0}$, such that

$$
f(x)=\sum_{k=0}^{\infty} b_{k}\left(x-x_{0}\right)^{k} \quad \text { for } \quad\left|x-x_{0}\right|<r .
$$

The biggest such $r$ is called the radius of convergence of the power series (8). By taking the $k$ th-order derivative and evaluating it at $x_{0}$ we find that $b_{k}=$ $\left(f^{(k)}\left(x_{0}\right)\right) / k$ ! so that (8) is a Taylor series. A $C^{\infty}$ function $f(x)$ is analytic near a point $x_{0}$ if and only if there exist positive constants $M_{0}, M$, and $\delta$ such that

$$
\left|f^{(k)}(x)\right| \leq M_{0} M^{k} k !, \quad\left|x-x_{0}\right| \leq \delta \quad \text { for all } k=0,1,2, \ldots
$$

Condition (9) is called the Cauchy estimates. If this condition is satisfied, then writing $f(x)=P_{n}(x)+R_{n+1}(x)$, where

$$
P_{n}(x)=\sum_{k=0}^{n} \frac{f^{(k)}\left(x_{0}\right)}{k !}\left(x-x_{0}\right)^{k}
$$

is the $n$th order Taylor polynomial and $R_{n+1}(x)$ is the remainder,

$$
R_{n+1}(x)=\frac{f^{n+1}\left(x_{*}\right)\left(x-x_{0}\right)^{n+1}}{(n+1) !} \text { for some } x_{*} \text { between } x_{0} \text { and } x,
$$

we can see that the remainder goes to zero as $n \rightarrow \infty$. This shows that $f(x)$ is equal to its Taylor series about $x_{0}$ for $\left|x-x_{0}\right|<r$ with $r=1 / M$.

This discussion tells us that to prove that the price-dividend function is analytic, it suffices to show that Cauchy estimates (9) for the price-dividend function hold. ${ }^{17}$ However, these estimates do not necessarily yield the radius of convergence. The most natural way to accomplish this is by the method of complexification, that is, in the price-dividend function we substitute the real variable $x$ with the complex variable $z=x+i y$.

When a function $f(x)$ is analytic, the power series representation of $f(x)$ (Equation (8)) can be complexified by replacing $x$ with $z=x+i y$ to obtain

$$
f(z)=\sum_{k=0}^{\infty} \frac{f^{(k)}\left(z_{0}\right)}{k !}\left(z-z_{0}\right)^{k} \quad \text { for } \quad\left|z-z_{0}\right|<r, \quad z_{0}=x_{0}+i 0 .
$$

${ }^{17} \mathrm{ACCH}$ (2003) provides these estimates. Also, Chen, Cosimano, and Himonas (2003) demonstrate how to obtain estimates for Campbell and Cochrane's (1999) asset pricing model. 
Here $f^{(k)}(z)$ denotes the $k$ th order complex derivative of $f(z)$. We mention that the usual calculus formulas for real differentiation hold true for complex differentiation too. Separating the function $f$ into its real and imaginary parts we write $f(z)=f_{1}(x, y)+i f_{2}(x, y)$. Then we have

$$
\frac{\partial f}{\partial x}=\frac{\partial f_{1}}{\partial x}+i \frac{\partial f_{2}}{\partial x}=\sum_{k=0}^{\infty} \frac{f^{(k)}\left(z_{0}\right)}{k !} k\left(z-z_{0}\right)^{k-1},
$$

while

$$
\frac{\partial f}{\partial y}=\frac{\partial f_{1}}{\partial y}+i \frac{\partial f_{2}}{\partial y}=\sum_{k=0}^{\infty} \frac{f^{(k)}\left(z_{0}\right)}{k !} i k\left(z-z_{0}\right)^{k-1} .
$$

This means that an analytic function must satisfy the pair of partial differential equations

$$
\frac{\partial f_{1}}{\partial x}=\frac{\partial f_{2}}{\partial y} \quad \text { and } \quad \frac{\partial f_{1}}{\partial y}=-\frac{\partial f_{2}}{\partial x} .
$$

These are called the Cauchy-Riemann equations (see Ahlfors (1979, p. 26)).

This leads to the following characterization: $f$ is said to be holomorphic (or analytic) in $D$ if it satisfies the Cauchy-Riemann equation

$$
\frac{\partial f}{\partial \bar{z}}=\frac{\partial f}{\partial x}+i \frac{\partial f}{\partial y}=0
$$

After complexifying the price-dividend function, we can identify the largest domain $D$ in the complex plane where the price-dividend function is (complex) analytic (or holomorphic). Finally, to find the radius of convergence of the Taylor series of the price-dividend function about a point $x_{*}$ we compute the distance of $x_{*}$ from the boundary of $D$.

THEOREM 1: The price-dividend function $P$ is real-analytic in the open interval $(-\infty, \infty)$. Moreover, its Taylor series expansion about a point $x_{*} \in(-\infty, \infty)$ has radius of convergence equal to $\infty$.

ProOF: Since $P$ satisfies the integral equation (6) and since by Proposition 1 it belongs to the space $\mathcal{S}$, to prove Theorem 1 it suffices to show that the function

$$
F(x)=\int_{-\infty}^{\infty} \exp \left[-\frac{1}{2 \sigma^{2}}[s-\psi(x)]^{2}\right][1+P(s)] d s
$$


is analytic in $(-\infty, \infty)$ and its Taylor series about any point has infinite radius of convergence, considering that $K_{0} \exp \left[K_{1} x\right]$ has the same properties. ${ }^{18}$ For this, in $F(x)$ we replace $x$ with $z=x+i y$ (i.e., we complexify) and we show that the integral expression defining $F(z)$,

$$
F(z)=\int_{-\infty}^{\infty} \exp \left[-\frac{1}{2 \sigma^{2}}[s-\psi(z)]^{2}\right][1+P(s)] d s,
$$

makes sense, and it is a holomorphic (or (complex) analytic) function on the entire complex plane. This follows immediately from the following more general result, applied with $f(z)=\psi(z)=\phi z+x_{0}+\sigma^{2}(1-\gamma)$ and $g(s)=1+P(s)$.

Q.E.D.

LEMMA 1: If $p$ is a positive number, $f(z)$ is a holomorphic function in the entire complex plane $\mathbb{C}$, and $g(t)$ is a measurable function such that

$$
|g(s)| \leq M_{0} \exp \left[k_{0}|s|\right], \quad s \in \mathbb{R},
$$

for some positive constants $M_{0}$ and $k_{0}$, then the formula

$$
F(z)=\int_{-\infty}^{\infty} \exp \left[-p[s-f(z)]^{2}\right] g(s) d s
$$

defines a holomorphic function in $\mathbb{C}$.

PROOF: We need to show that $F(z)$ has continuous first-order partial derivatives which satisfy the Cauchy-Riemann equations

$$
\frac{\partial F}{\partial x}+i \frac{\partial F}{\partial y}=0
$$

Let $z_{0}=x_{0}+i y_{0}$ be a fixed point in $\mathbb{C}$. Since $f(z)$ is bounded on any compact subset of $\mathbb{C}$, we have that there exists $M>0$ depending on $z_{0}$ such that

$$
\left|\exp \left[-p[s-f(z)]^{2}\right]\right| \leq M \exp \left[-(p / 2) s^{2}\right], \quad s \in \mathbb{R}, \quad\left|z-z_{0}\right| \leq 1 .
$$

Similarly, using the formulas

$$
\frac{\partial}{\partial x} \exp \left[-p[s-f(z)]^{2}\right]=2 p[s-f(z)] \frac{\partial f}{\partial x}(z) \exp \left[-p[s-f(z)]^{2}\right]
$$

and

$$
\frac{\partial}{\partial y} \exp \left[-p[s-f(z)]^{2}\right]=2 p[s-f(z)] \frac{\partial f}{\partial y}(z) \exp \left[-p[s-f(z)]^{2}\right]
$$

${ }^{18}$ The sum and product of analytic functions are analytic. See Rudin (1974). 
we obtain that

$$
\begin{aligned}
& \left|\frac{\partial}{\partial x} \exp \left[-p[s-f(z)]^{2}\right]\right| \leq M \exp \left[-(p / 2) s^{2}\right] \quad \text { and } \\
& \left|\frac{\partial}{\partial y} \exp \left[-p[s-f(z)]^{2}\right]\right| \leq M \exp \left[-(p / 2) s^{2}\right], \quad s \in \mathbb{R}, \quad\left|z-z_{0}\right| \leq 1,
\end{aligned}
$$

if we adjust the constant $M$. Using inequality (16) and our hypothesis that $g(s)$ satisfies (13), we obtain that $F(z)$ is a well-defined function for all $z \in \mathbb{C}$. Also, using inequalities (17) and the DCT (see Folland (1984, pp. 53-54)) we obtain that the partial derivatives of $F(z)$ with respect to $x$ and $y$ are well defined near every point $z_{0} \in \mathbb{C}$, are continuous, and that we can pass the differentiation inside the integral sign. Since $\exp \left[-p[s-f(z)]^{2}\right]$ is holomorphic, it satisfies the Cauchy-Riemann equations

$$
\left(\frac{\partial}{\partial x}+i \frac{\partial}{\partial y}\right) \exp \left[-p[s-f(z)]^{2}\right]=0
$$

and we have that

$$
\begin{aligned}
\left(\frac{\partial}{\partial x}+i \frac{\partial}{\partial y}\right) F(z) & =\int_{-\infty}^{\infty}\left(\frac{\partial}{\partial x}+i \frac{\partial}{\partial y}\right) \exp \left[-p[s-f(z)]^{2}\right] g(s) d s \\
& =\int_{-\infty}^{\infty} 0 \cdot g(s) d s=0
\end{aligned}
$$

which proves (15). This completes the proof of Lemma 1.

Q.E.D.

\subsection{The Analytic Method for Computing the Price-Dividend Function}

By Theorem 1 the price-dividend function, $P(x)$, is analytic and its Taylor series about every point has infinite radius of convergence. In the integral equation (6) there is a common term $\exp \left[K_{1} x\right]$ that captures the role of expected dividend growth, $x_{0}+\phi x$, on the price-dividend ratio. ${ }^{19}$ It is more convenient to compute the Taylor series for

$$
Q(x)=\exp \left[-K_{1} x\right] P(x)
$$

instead of $P(x)$. We refer to $Q(x)$ as the price-expected-dividend function. The function $Q(x)$ has the same analytic properties as the price-dividend function, since $\exp \left[-K_{1} x\right]$ is also real-analytic in the open interval $(-\infty, \infty)$.

\footnotetext{
${ }^{19}$ Strictly speaking $K_{0}$ includes the impact of the constant in expected dividend growth, $x_{0}$.
} 
If we replace the price-dividend-function, $P(x)$, with the price-expecteddividend function, $Q(x)$, in the integral equation (6), then it satisfies the integral equation

$$
Q(x)=K_{0}+\frac{K_{3} \exp \left[\phi K_{1} x\right]}{\sqrt{2 \pi} \sigma} \int_{-\infty}^{\infty} Q(s) \exp \left[-\frac{1}{2 \sigma^{2}}[s-(\phi x+\theta)]^{2}\right] d s,
$$

where $K_{3}=K_{0} \exp \left(x_{0} K_{1}+\left(\sigma^{2}(1-\gamma)^{2}(\phi-\alpha)(2+\phi-\alpha)\right) / 2\right)$. Since the price-expected-dividend function is analytic, we may express $Q(x)$ as a Taylor series

$$
Q(x)=\sum_{k=0}^{\infty} \frac{Q^{(k)}\left(x_{*}\right)}{k !}\left(x-x_{*}\right)^{k}, \quad x \in(-\infty, \infty) .
$$

Here $x_{*}$ is the average value of dividend growth.

Motivated by (20), we approximate the price-expected-dividend function numerically by a polynomial of the form

$$
Q_{n}^{c}(x)=\sum_{k=0}^{n} b_{k}\left(x-x_{*}\right)^{k} .
$$

Substituting this polynomial into the integral equation (19), computing the resulting integrals, and equating the coefficients of the same power of $x$ yields the following system of linear equations in the unknown coefficients

$$
\begin{aligned}
& b_{0}=K_{0}+K_{6} \sum_{k=0}^{n} b_{k} \sum_{i=0}^{k}\left(\begin{array}{c}
k \\
i
\end{array}\right) w_{i, k}^{(0)}\left(x_{*}\right) a_{i} \text { and } \\
& b_{l}=K_{6} \sum_{k=0}^{n} b_{k} \sum_{i=0}^{k}\left(\begin{array}{c}
k \\
i
\end{array}\right) \frac{1}{l !} w_{i, k}^{(l)}\left(x_{*}\right) a_{i}
\end{aligned}
$$

for $l=1, \ldots, n$. Here, $K_{6}=\frac{K_{0}}{\sqrt{\pi}} \exp \left(\frac{\sigma^{2}}{2}\left[1-\gamma+K_{1}\right]^{2}-\frac{\sigma^{2}}{2}[1-\gamma]^{2}\right), w_{i, k}(x)=$ $\exp \left[K_{1}\left(x_{0}+\phi x\right)\right]\left(x_{0}-x_{*}+\phi x\right)^{k-i}$, and $a_{i}=\sum_{j=0}^{i}\left(\begin{array}{c}i \\ j\end{array}\right)\left(\sigma^{2}\left[1-\gamma+K_{1}\right]\right)^{i-j} \times$ $(\sqrt{2} \sigma)^{j}\left(1+(-1)^{j}\right)(1 / 2) \Gamma\left[\frac{j+1}{2}\right]$. Solving (22) we find the coefficients $b_{k}$ in our approximate solution (21), which allows us to compute the price-dividend function using (18) by $P_{n}^{c}(x)=\exp \left[K_{1} x\right] Q_{n}^{c}(x)$.

\subsection{Error Analysis for the Computed Price-Dividend Function}

We now consider the error in our approximate solution (21) for the priceexpected-dividend function

$$
\text { error }=\max _{\left|x-x_{*}\right|<\mu r}\left|Q(x)-Q_{n}^{c}(x)\right| .
$$


Here $r>0$ and $0<\mu<1$ are to be chosen later. Write the price-expecteddividend, $Q(x)$, as

$$
Q(x)=Q_{n}(x)+R_{n}(x) .
$$

Here $Q_{n}(x)=\sum_{k=0}^{n} \frac{Q^{(k)}\left(x_{*}\right)}{k !}\left(x-x_{*}\right)^{k}$ and $R_{n}(x)=\sum_{k=n+1}^{\infty} \frac{Q^{(k)}\left(x_{*}\right)}{k !}\left(x-x_{*}\right)^{k}$. Consequently, the error may be decomposed into two parts based on

$$
\left|Q(x)-Q_{n}^{c}(x)\right| \leq\left|R_{n}(x)\right|+\left|Q_{n}^{c}(x)-Q_{n}(x)\right| .
$$

We call the first error the analytic error and the second part the computational error.

Using integral equation (19) and the Cauchy integral formula we obtain the following estimate for the derivatives of the price-expected-dividend function $^{20}$ :

$$
\left|Q^{(k)}\left(x_{*}\right)\right| \leq B_{r} \frac{k !}{r^{k}} .
$$

Here $B_{r}$ is a fixed positive number derived in the Appendix. For the parameters used here we find that $B_{r}=637.5601$ for the Mehra-Prescott case and $B_{r}=2269.1687$ for the external habit case when $r=20 \sigma$. So if $\left|x-x_{*}\right|<\mu r$, $0<\mu<1$, then

$$
\left|R_{n}(x)\right| \leq \sum_{k=n+1}^{\infty} \frac{\left|Q^{(k)}\left(x_{*}\right)\right|}{k !}\left(\frac{\left|x-x_{*}\right|}{r}\right)^{k} r^{k} \leq B_{r} \frac{\mu^{n+1}}{1-\mu} .
$$

Using this estimate for the analytic error we see that

$$
\text { error } \leq B_{r} \frac{\mu^{n+1}}{1-\mu}+\max _{\left|x-x_{*}\right|<\mu r}\left|Q_{n}^{c}(x)-Q_{n}(x)\right| .
$$

In the Mehra-Prescott case the analytic error is below $\epsilon / 2$ when we consider only $\left|x-x_{*}\right|=.3 \sigma$ for $n=9$ so that $\mu=.015 ; \epsilon$ is a machine epsilon number. In this paper a number is called machine epsilon when it is less than $10^{-16}$. However, we can consider a larger domain for the price-expected-dividend function of $\left|x-x_{*}\right|=8.6 \sigma$ by using $n=50$ and $\mu=.43$, and still keep the analytic error below $\epsilon / 2$. The results are similar for the external habit case. Therefore, we can make the analytic error less than $\epsilon / 2$ by considering a smaller disk of radius $\mu r$ or by adding sufficiently many terms to the Taylor series (21).

The computation error for the price-expected-dividend function in (25) is more involved. As new coefficients are added to the computational solution, $Q_{n}^{c}(x)$, the original coefficients can change since all new and old

\footnotetext{
${ }^{20}$ See Ahlfors (1979, p. 120) or Conway (1973, Corollary 5.9). For an application to Taylor series, see Weinberger (1965, pp. 264-267).
} 
coefficients are involved in system (22). This computation error can be estimated, but it is quite tedious. The basic idea is as follows: Put the power series (20) for $Q(x)$ into the integral equation (19). Manipulate this expression following the same procedures used in the derivation of (22). These calculations result in an infinite system of equations in the coefficients for the power series (20) of $Q(x)$, i.e., $\frac{Q^{(k)}\left(x_{*}\right)}{k !}$. Equation (23) provides estimates for these coefficients. By applying Gaussian elimination to the first $n$ equations and coefficients in this infinite system of equations, we can express the errors in first $n$ coefficients in the following form

$$
b_{j}-\frac{Q^{(j)}\left(x_{*}\right)}{j !}=E_{j}\left(\frac{Q^{(k)}\left(x_{*}\right)}{k !} ; k \geq n+1\right) \text { for } j=0, \ldots, n .
$$

The errors, $E_{j}$, are functions that are linear combinations of the higher-order coefficients, $\frac{Q^{(k)}\left(x_{*}\right)}{k !}$ for $k \geq n+1$. These errors tend to zero as the number of coefficients increases, since the bounds (23) tend to zero as more coefficients are added. Thus, we can identify when the computational error tends to machine epsilon based on the bounds (23).

In our program, we stop adding coefficients when $\sup _{i=0,1, \ldots, n-1}\left|b_{n-1, i}-b_{n, i}\right|<$ $\epsilon /(2 n)$, where $b_{n, i}$ is the $i$ th coefficient of the computed solution (21) with $n$ coefficients. We take the stability of the coefficients as an indication that the coefficients are sufficiently close to the true Taylor series coefficients. This rule is satisfied for $n=9$ coefficients in the Mehra-Prescott case and $n=11$ in the external habit case. The change in the early coefficients disappears as more coefficients are added. To be complete, we increased the number of coefficients in our numerical procedure to 50 and do not see any change in the early coefficients greater than $\epsilon /(2 n)$. Thus, we know how to make the analytic error and the computational error less than $\epsilon / 2$, so that we have a well-defined numerical procedure to approximate the analytic solution to the price-dividend function with error less than machine $\epsilon$.

\section{COMPARISON OF ANALYTIC SOLUTION WITH NUMERICAL APPROXIMATION}

We parameterize the asset pricing model in the same way as found in the literature. This allows us to compute (21), which yields the price-dividend function with error given by $(25) .{ }^{21}$ We can also compare our solution to a Gaussian quadrature procedure (see Judd (1998, pp. 259-263)) to see how it fares against that technique. We use a weighted sum of Chebyshev polynomials to represent the solution to (6) in the interval $(-\bar{x}, \bar{x})$, where $\bar{x}$ is a multiple

\footnotetext{
${ }^{21}$ In the calculations we use this error relative to the computed solution for the price-dividend function at the average value of dividend growth to make the comparison between solutions consistent.
} 
of the standard deviation for dividend growth. In addition we have to evaluate the integral in (6). To do this we use the Gaussian quadrature weights and nodes, which give us an approximation for this integral using $n$ nodes. ${ }^{22}$ We use a collocation method to solve for the weights for the Chebyshev polynomials for the proposed solution. This involves solving for the roots of the $n$th order Chebyshev polynomial which are the optimal points to use. The measure, used to determine the best number of nodes to calculate, is the residual from the Euler equation divided by the price-dividend function at the average value of dividend growth. We find that this residual is always below machine zero for 15 nodes. $^{23}$

\subsection{The Mehra and Prescott Case}

We parameterize the Mehra and Prescott case as in Lucas (1994). The parameter values are $\sigma=.036, \gamma=2.5, \beta=.95, x_{*}=.017$, and $\phi=-.14$. With this parameterization, we find that the supremum of the changes in the coefficients is machine epsilon when we include 9 coefficients. We report in Table I the moments, means, variances, and relative error for the price-dividend ratio we generate from this model and compare them to those found using the Gaussian quadrature method. We solve (6) for the price-dividend function with the numerical method by bounding the error term with 3, 4, and 5 standard deviations to the right and left of the mean. ${ }^{24}$

We see in Table I that the moments generated by Gaussian quadrature methods come close to matching the moments from our solution. ${ }^{25}$ We run the

\footnotetext{
${ }^{22}$ We use the procedure GaussianQuadratureWeights in Mathematica to obtain the weights and nodes.

${ }^{23}$ For the arguments in favor of using quadrature procedures in asset pricing, see Tauchen and Hussey (1991). Since we are using a normal distribution, we could use Gauss-Hermite quadrature methods, which allow integration over the entire support of the distribution. We choose Gaussian quadrature instead because in several asset pricing models, such as Abel's internal habit persistence, one cannot use the entire support of the distribution. Dividend growth must be bounded from above and below. With the Gaussian quadrature procedure, we can bound the support of the distribution. Second, even if there were no bounds on the support of the distribution, we could still get an accurate solution with the Gaussian quadrature procedure by integrating over many standard deviations as we can make the limits of integration as large as we like. Last, in order to approximate the $P(x)$ function with Chebyshev polynomials, we need a maximum and minimum growth rate for the dividend and, consequently, we need to bound the support of the distribution.

${ }^{24}$ The reason we do this is because, for many models, bounds on the distribution of the random variable arise naturally from the model as for example the habit persistence model in Abel (1990). This gives us an idea of the accuracy of the solution and how such judgments affect the solution.

${ }^{25} \mathrm{ACCH}$ (2003) provides complete details concerning the coefficients and errors. ACCH also compares the analytic solution with Lucas' (1994) and finds the moments comparable. We can obtain all these moments from the price-dividend ratio, the bond price converting them to returns, and the interest rate and then integrating over the random variable to obtain expected values and standard deviations.
} 
TABLE I

COMPARISON OF RESULTS ${ }^{\mathrm{a}}$

\begin{tabular}{lcccr}
\hline \hline Statistic & $\begin{array}{c}\text { Analytic Solution } \\
\text { Nine Coefficients }\end{array}$ & $\begin{array}{c}\text { Gaussian } \\
\text { Three Std. }\end{array}$ & $\begin{array}{c}\text { Gaussian } \\
\text { Four Std. }\end{array}$ & $\begin{array}{r}\text { Gaussian } \\
\text { Five Std. }\end{array}$ \\
\hline$E\left(R_{s}\right)$ & .098 & .098 & .098 & .098 \\
$\sigma\left(R_{s}\right)$ & .046 & .052 & .046 & .046 \\
$E\left(R_{B}\right)$ & .094 & .094 & .094 & .094 \\
$\sigma\left(R_{B}\right)$ & .014 & .014 & .014 & .014 \\
$E\left(R_{s}-R_{B}\right)$ & .004 & .004 & .004 & .004 \\
$\sigma\left(R_{s}-R_{B}\right)$ & .044 & .044 & .044 & .044 \\
$E\left(C_{t+1}\right) / C_{t}$ & .017 & .017 & .017 & .017 \\
$\sigma\left(\left(C_{t+1}\right) / C_{t}\right)$ & .036 & .036 & .036 & .036 \\
$P(.017)$ & 12.73 & 12.26 & 12.72 & 12.73 \\
Error & $\epsilon$ & $-4.45 \times 10^{-16}$ & $1.40 \times 10^{-16}$ & $\epsilon$ \\
\hline
\end{tabular}

a Notation: $R_{S}$ is the return to the stock and $R_{B}$ is the return to the bond. $C_{t}$ is per capita consumption at time $t$ and $P$ is the price-dividend ratio. $E$ represents an expectation and $\sigma$ is a standard deviation. $P(.017)$ is the value of the price dividend ratio at the historic average consumption growth. All the evaluations of the statistics with the analytic solution are at the historic average consumption growth, .017. The parameter values used are $\beta=$ $.95, \sigma=.036, \gamma=2.5, x_{*}=.017$, and $\phi=-.14$. We obtain these moments by using the price-dividend ratio and the price of the bond, convert them to returns, and then integrate to obtain the expected returns and standard deviations. The $\epsilon$ in the error row means that the relative error is below $10^{-16}$.

Gaussian quadrature procedure with three different bounds on the error term: 3,4 , and 5 standard deviations. One reason we do this is that when thinking of a normal distribution, it seems reasonable that integrating over 3 standard deviations will give an accurate answer to any problem since $99 \%$ of the probability lies within the 3 standard deviations. However, we will see for the asset pricing results here it is not the case and we need 5 standard deviations.

We see in Table I that when using 3 standard deviations, the moments match the analytic solution at the historic value for dividend growth, but the pricedividend ratio is off by 47 cents. This is a large deviation from the truth since that large a difference in an asset price generated by the model can change an investor's decision. Suppose that the dividend today is $\$ 1.00$ and one was looking to find a mispriced security to buy or sell off. Suppose that the security is trading at $\$ 12.50$. Then one would sell or even sell short the security according to the numerical solution, while one would buy the security according to the analytic solution. This is of interest since the relative error to that numerical procedure in the last row of Table I, measured as the deviation of the Euler equation from zero divided by the price-dividend ratio, is very small and one may conclude that this solution is accurate. We do see, however, that as we increase the number of standard deviations over which we integrate, the price-dividend ratio is the same as the analytic solution in Table I at the historic average.

It is instructive to note that while in this case we can obtain a very good solution with the numerical method, the analytic solution gives the precise results 
with an actual error term as to how much the price-dividend ratio may be off from the truth within the radius of convergence. One cannot provide this with the numerical methods. Second, except for algebraic errors, there are really no errors to be made as judgment errors. That is, once we have the solution and an estimate of the bound on the remainder term, then the procedure is straightforward and we know we will have the solution. An exception to this is the result of Santos (2000) who finds under certain conditions, the residual on the Euler equation is of the same order of magnitude as the error in the policy function. Even there, however, when estimating the policy function numerically, one still needs to decide which numerical procedure to use. Consequently, one still needs to use good judgment in order to obtain an accurate solution.

\subsection{Abel's Habit Persistence Cases}

In ACCH (2003) we report a detailed comparison with all the habit persistence cases of Abel (1990). Here we focus on the external habit case with parameters $\phi=-.14, \beta=.95, \gamma=2.5, \alpha=1, \rho=0$, and $x_{*}=.017$, the parameterization of Lucas (1994) with the exception that we will be looking at the relative consumption model, $\alpha=1$ and $\rho=0$. With this parameterization, we find that the supremum of the changes in the coefficients is less than $\frac{\epsilon}{2 n}$ when we include $n=11$ coefficients.

Once again when we solve for the model using the Gaussian quadrature procedure with 5 standard deviations, we obtain the same solution at the point $x_{*}$ in Table II. One interesting result is that with 4 standard deviations, all the moments are matched with the analytic solution except for the price-dividend ratio. In particular, from the error in this procedure, the Euler equation residual divided by the price-dividend ratio, one would conclude that the solution is very accurate with a 4 standard deviation bound; however, in terms of pricing, the solution is wrong by three cents per dollar of dividend.

The comparison between the analytic solutions to these asset pricing models and their numerical solution points to some benefits of solving for the analytic solution. First, the relative error term, the approximate Euler equation divided by the approximate price-dividend function, used in the Gauss quadrature solution does not necessarily reflect the accuracy of the price-dividend function. In addition, our procedure identifies the exact conditions when the price-dividend function is well defined and accurate so that no judgment is necessary in assessing our price-dividend function.

\section{CONCLUSION}

In this paper we present a new method for solving asset pricing models of one state variable which yields an analytic price-dividend function, and we give a detailed analysis for both Mehra and Prescott's and Abel's external habit models (see Theorem 1). Recall that a function is analytic in an open interval if it 
TABLE II

COMPARISON OF RESUltS: RELATIVE CONSUMPTION, $\alpha=1, \rho=0^{\mathrm{a}}$

\begin{tabular}{lcccc}
\hline \hline Statistic & $\begin{array}{c}\text { Analytic Solution } \\
\text { Eleven Coefficients }\end{array}$ & $\begin{array}{c}\text { Gaussian } \\
\text { Three Std. }\end{array}$ & $\begin{array}{c}\text { Gaussian } \\
\text { Four Std. }\end{array}$ & $\begin{array}{c}\text { Gaussian } \\
\text { Five Std. }\end{array}$ \\
\hline$E\left(R_{s}\right)$ & .075 & .075 & .075 & .75 \\
$\sigma\left(R_{s}\right)$ & .094 & .091 & .094 & .094 \\
$E\left(R_{B}\right)$ & .069 & .068 & .069 & .069 \\
$\sigma\left(R_{B}\right)$ & .071 & .070 & .071 & .071 \\
$E\left(R_{s}-R_{B}\right)$ & .006 & .007 & .006 & .006 \\
$\sigma\left(R_{s}-R_{B}\right)$ & .0844 & .0835 & .0844 & .0844 \\
$E\left(C_{t+1}\right) / C_{t}$ & .017 & .017 & .017 & .017 \\
$\sigma\left(\left(C_{t+1}\right) / C_{t}\right)$ & .036 & .036 & .036 & .036 \\
$P(.017)$ & 19.03 & 18.05 & 19.00 & 19.03 \\
Error & $\epsilon$ & $-1.96 \times 10^{-16}$ & $\epsilon$ & $\epsilon$ \\
\hline
\end{tabular}

a Notation: $R_{S}$ is the return to the stock and $R_{B}$ is the return to the bond. $C_{t}$ is per capita consumption at time $t$ and $P$ is the price-dividend ratio. $E$ represents an expectation and $\sigma$ is a standard deviation. $P(.017)$ is the value of the price-dividend ratio at the historic average consumption growth. All the evaluations of the statistics with the analytic solution are at the historic average consumption growth, .017 . The parameter values used are $\beta=.95, \sigma=.036, \gamma=2.5, x_{*}=.017$, and $\phi=-.14$. We obtain these moments by using the price-dividend ratio and the price of the bond, convert them to returns, and then integrate to obtain the expected returns and standard deviations. The $\epsilon$ in the error row means that the relative error is below $10^{-16}$.

can be expressed as a convergent power series near every point of that interval. In Chen, Cosimano, and Himonas (2003) this method is extended further to show that the price-dividend function from Campbell and Cochrane's (1999) model is analytic as long as their sensitivity function is holomorphic. Also, Chen, Cosimano, and Himonas are currently exploring the extent to which this method can be extended to the higher-dimensional Campbell and Cochrane model developed by Wachter (2002). We think this procedure can be used to handle more complicated asset pricing models in one as well as in several dimensions.

To establish an analytic solution to an asset pricing model, we first need an increasing, concave utility function (1), which becomes holomorphic after complexification. We also need a bijective, holomorphic stochastic process for the state variable (2) in a neighborhood of zero after it is also complexified so that the stochastic process for the future state variable may be inverted. This leads to an integral equation for the price-dividend function that, after making an appropriate change of variables, depends holomorphically on the state variable near zero. Finally, after establishing that the solution is integrable, one expects the complex analysis (in one or several variables) similar to that presented here to go through to obtain the analyticity of the price-dividend function near zero. The formulation of general conditions under which the price-dividend function is holomorphic is the main subject of our research. 
The analyticity property of the price-dividend function has motivated a numerical procedure implementing higher-order Taylor approximation with error measured by using Cauchy's integral formula. The results obtained this way are compared with a state of the art numerical procedure from the extant literature.

There are several directions for future research to extend this method. First, increase the dimension of the asset pricing problems. There are several problems of interest in multidimensional cases such as portfolio choice over many assets. Second, consider stochastic growth models, which yield nonlinear integral equations. Jin and Judd (2002), and Schmitt-Grohe and Uribe (2004) examine both extensions for the perturbation method, and it would be interesting to extend our method to those cases. Finally, we showed in ACCH (2003) how accurate solutions to the asset pricing models can be used to accurately price European call options in circumstances more general than in the Black and Scholes model.

Dept. of Mathematics, Eastern Michigan University, Ypsilanti, MI 48197, U.S.A; ocalin@emunix.emich.edu.,

Dept. of Mathematics, Idaho State University, Pocatello, ID 83209, U.S.A; chenyu@isu.edu,

Dept. of Finance, Mendoza College of Business, University of Notre Dame, Notre Dame, IN 46556, U.S.A.; cosimano.1@nd.edu; www.nd.edu/ tcosiman/, and

Dept. of Mathematics, University of Notre Dame, Notre Dame, IN 46556, U.S.A.; Alex.A.Himonas.1@nd.edu.

Manuscript received June, 2003; final revision received November, 2004.

\section{REFERENCES}

ABEL, A. B. (1990): "Asset Prices under Habit Formation and Catching up with the Jones," American Economic Review, 80, 38-42.

AhLFORS, L. V. (1979): Complex Analysis (3rd ed.). New York: McGraw-Hill.

AltuĞ, S., AND P. LABADIE (1994): Dynamic Choice and Asset Markets. New York: Academic Press.

Athanasoulis, S. G., O. L. Calin, T. F. Cosimano, AND A. A. Himonas (2003): "Solving Asset Pricing Models when the Price-Dividend Function Is Analytic," Working Paper, University of Notre Dame.

ATHANASOUlis, S. G., AND R. J. SHILleR (2001): "World Income Components: Measuring and Exploiting Risk-Sharing," American Economic Review, 91, 1031-1054.

Birdarkota, P. V., AND J. H. MCCulloch (2003): "Consumption Asset Pricing with Stable Shocks-Exploring a Solution and Its Implications for Mean Equity Returns," Journal of Economic Dynamics and Control, 27, 399-422.

Blume, L., D. EASLEY, AND M. O'HARA (1982): “Characterization of Optimal Plans for Stochastic Dynamic Programs," Journal of Economic Theory, 28, 221-234.

BuRnSIDE, C. (1998): "Solving Asset Pricing Models with Gaussian Shocks," Journal of Economic Dynamics and Control, 22, 329-340. 
CAmpBell, J. Y. (1986): "Bond and Stock Returns in a Simple Exchange Model,” Quarterly Journal of Economics, 101, 785-803.

(2001): "Consumption-Based Asset Pricing," in Handbook of the Economics of Finance, ed. by G. M. Constantinides, M. Harris, and R. Stulz. Amsterdam: North-Holland, 803-887.

CAMPBELl, J. Y., AND J. Cochrane (1999): "By Force of Habit, A Consumption-Based Explanation of Aggregate Stock Market Behavior," Journal of Political Economy, 107, 205-251.

CecchetTi, S. G., P. LAM, AND N. MARK (1990): "Mean Reversion in Equilibrium Asset Prices," American Economic Review, 80, 398-418.

(2000): "Asset Prices with Distorted Beliefs: Are Equity Returns Too Good to Be True?" American Economic Review, 90, 787-805.

Chen, Y., T. F. Cosimano, AND A. A. Himonas (2003): "By Force of Habit: An Exploration of Asset Pricing Models Using Analytic Methods," Working Paper, University of Notre Dame.

COLLARD, F., AND M. JUILLARD (2001): "Accuracy of Stochastic Perturbation Methods: The Case of Asset Pricing Models," Journal of Economic Dynamics and Control, 25, 979-999.

Constantinides, G. M., AND D. DufFIE (1996): "Asset Pricing with Heterogenous Consumers," Journal of Political Economy, 104, 219-240.

Conway, J. B. (1973): Functions of One Complex Variable, Graduate Texts in Mathematics, Vol. 11. New York: Springer-Verlag.

ENGLE, R. F. (1982): "Autoregressive Conditional Heteroscedasticity with Estimates of the Variance of United Kingdom Inflation," Econometrica, 50, 987-1007.

EPSTEIN, L. G., AND S. E. ZIN (1989): "Substitution, Risk Aversion, and the Temporal Behavior of Consumption and Asset Returns: A Theoretical Framework," Econometrica, 57, 937-968.

(1990): "First-Order Risk Aversion and the Equity Premium Puzzle," Journal of Monetary Economics, 26, 387-407.

(1991): "Substitution, Risk Aversion, and the Temporal Behavior of Consumption and Asset Returns: An Empirical Analysis," Journal of Political Economy, 99, 263-286.

FolLAND, G. B. (1984): Real Analysis. New York: John Wiley \& Sons.

JIN, H., AND K. L. JUDD (2002): "Perturbation Methods for General Dynamic Stochastic Models," Working Paper, Stanford University.

JUDD, K. L. (1996): "Approximation, Perturbation, and Projection Methods in Economic Analysis," in Handbook of Computational Economics, ed. by H. M. Amman, D. A. Kendrick, and J. Rust. Amsterdam: Elsevier, 511-585.

(1998): Numerical Methods in Economics. Cambridge, MA: MIT Press.

JUDD, K. L., AND S. GUU (2001): "Asymptotic Methods for Asset Market Equilibrium Analysis," Economic Theory, 18, 127-157.

KAPLAN, W. (1956): Advanced Calculus (3rd Printing). Reading, MA: Addison-Wesley.

LABADIE, P. (1989): "Stochastic Inflation and the Equity Premium," Journal of Monetary Economics, 24, 277-298.

LUCAS, D. J. (1994): “Asset Pricing with Undiversifiable Income Risk and Short Sales Constraints Deepening the Equity Premium Puzzle," Journal of Monetary Economics, 34, 325-341.

LuCAS, R. E., JR. (1978): “Asset Prices in a Pure Exchange Economy," Econometrica, 46, $1429-1445$.

Mehra, R., And E. C. Prescott (1985): “The Equity Premium: A Puzzle,” Journal of Monetary Economics, 15, 145-161.

(2003): "The Equity Premium in Retrospect," in Handbook of the Economics of Finance, ed. by G. M. Constantinides, M. Harris, and R. Stulz. Amsterdam: North-Holland, 889-938.

Rudin, W. (1974): Real and Complex Analysis. New York: McGraw-Hill.

SANTOS, M. S. (1991): "Smoothness of the Policy Function in Discrete Time Economic Models," Econometrica, 59, 1365-1382.

(1992): "Differentiability and Comparative Analysis in Discrete-Time-Infinite-Horizon Optimization," Journal of Economic Theory, 57, 222-229. 565-570. 
(1994): "Smooth Dynamics and Computation in Models of Economic Growth," Journal of Economic Dynamics and Control, 18, 879-895.

(1999): "Numerical Solution of Dynamic Economic Models," in Handbook of Macroeconomics, ed. by J. B. Taylor and M. Woodford. Amsterdam: Elsevier, 311-382.

(2000): "Accuracy of Numerical Solutions Using the Euler Equation Residuals," Econometrica, 68, 1377-1402.

SCHMITT-Grohe, S., AND M. URIBE (2004): "Solving Dynamic General Equilibrium Models Using a Second Order Approximation to the Policy Function," Journal of Economic Dynamics and Control, 28, 755-775.

TAUCHEN, G., AND R. HusSEY (1991): "Quadrature-Based Methods for Obtaining Approximate Solutions to the Integral Equations of Nonlinear Rational Expectations Models," Econometrica, 59, 371-396.

Tsionas, E. G. (2003): "Exact Solution of Asset Pricing Models with Arbitrary Shock Distributions," Journal of Economic Dynamics and Control, 27, 843-851.

WACHTER, J. A. (2002): "Habit Formation and Returns on Bonds and Stocks," Working Paper, New York University.

WANG, J. (1994): “A Model of Competitive Stock Trading Volume,” Journal of Political Economy, $102,127-168$.

WeInBERger, H. F. (1965): A First Course in Partial Differential Equations. New York: John Wiley \& Sons. 